\title{
Segmentação da demanda dos planos e seguros privados de saúde: uma análise das informações da PNAD/98
}

\author{
Segmentation of the demand of the plans and private \\ insurances of health: an analysis of the information \\ of PNAD/98
}

Ligia Bahia ${ }^{1}$

Antonio José Leal Costa 1

Cristiano Fernandes 2

Ronir Raggio Luiz ${ }^{1}$

Maria de Lourdes T. Cavalcanti 1

1 Núcleo de Estudos de Saúde Coletiva da Universidade Federal do Rio de Janeiro. Av. Brigadeiro Trompowsky $\mathrm{s} / \mathrm{n}$, Ala Sul, 5 o andar, Hospital Universitário. Ilha do Fundão 21941-000 Rio de Janeiro RJ. ligiabahia@terra.com.br 2 Departamento de Engenharia Elétrica da Pontifícia Universidade Católica do Rio de Janeiro.
Abstract The authors investigate the extent to which adverse selection and moral hazard influence private health insurance demand in Brazil, based on the 1998 National Socioeconomic and Health Survey. Three population groups were compared, namely the one whose private health insurance was acquired by their employers, a second group whose insurance was individually acquired directly from insurance providers, and finally the one without any type of private health insurance and exclusively assisted by public health services. A taxonomy of health insurance plans was developed, and other covariates were also considered in the analysis, namely health states, health care services use, and health insurance coverage. The results suggest the occurrence of both types of market failures. Private health insurance coverage was found to be associated with unfavorable selfreported health states while the type of coverage appears to be associated with a more frequent use of health services. Multivariate logistic regression analyses, with multiple controls, showed that only health care expenditures remained associated with both adverse selection and moral hazard. Although a clear cut pattern of asymmetric information was not observed, results point out the need to further investigate the relations among morbidity, health care services use, health care expenditures and the varying types of health insurance plans. Key words Private health plans, Market failures, Asymmetric information
Resumo Este artigo apresenta uma investigação preliminar da presença da seleção adversa e do risco moral (moral hazard) na demanda ativa por planos de saúde no Brasil, a partir dos dados da PNAD/98. O presente estudo compara indivíduos cujas coberturas decorrem do vínculo de trabalho, com aqueles cujos planos resultam de uma demanda individual às empresas que os comercializam e os que não têm acesso a esquemas assistenciais alternativos ao SUS. A elaboração de uma tipologia de planos de saúde, combinada com variáveis relacionadas com as condições de saúde, utilização de serviços e gastos com saúde, sugere a existência de falhas de mercado. A percepção de uma condição de saúde mais desfavorável parece estar associada à busca de cobertura e o tipo de cobertura com o maior uso de serviços de saúde. Quando analisadas através de dois modelos de regressão logística com múltiplos controles, onde a variável de desfecho é indicadora de seleção adversa ou moral hazard, essas diferenças se atenuam, com exceção dos gastos com saúde. Os resultados não evidenciam uma inquestionável assimetria de informações, mas sinalizam a necessidade de aprofundar o conhecimento sobre as relações entre morbidade, utilização de serviços de saúde, gastos com saúde e tipo de cobertura.

Palavras-chave Planos privados de saúde, Falhas de mercado, Assimetria de informação 


\section{Introdução}

O Suplemento Saúde da Pesquisa Nacional por Amostra de Domicílios, conduzida pelo Instituto Brasileiro de Geografia e Estatística em 1998 (PNAD/98), é o primeiro levantamento de base populacional com abrangência nacional sobre cobertura de planos e seguros privados de saúde. Essa pesquisa, além de permitir uma estimativa do contingente de pessoas vinculadas ou não aos planos de saúde, traz informações sobre outros aspectos relevantes da saúde dos indivíduos, tais como morbidade percebida e referida, gastos em bens e serviços de saúde, etc. A partir das questões incluídas no seu corpo principal, são coletadas na PNAD outras informações sobre emprego, renda e educação, permitindo assim o estudo de inúmeras associações entre a percepção sobre a morbidade, utilização de serviços de saúde, condições de cobertura e status sociocupacional.

Este trabalho, contudo, prioriza determinados aspectos diretamente envolvidos no debate sobre a regulamentação das operadoras de planos e seguros de saúde. Seu objetivo é o exame da presença de dois mecanismos, indicativos das falhas de mercado dos seguros, a seleção adversa e o moral hazard, que, entre outros fatores, justificaram a recente regulação governamental deste segmento, consubstanciada na lei 9.656-98 e seus sucedâneos. Para analisar os mecanismos sugestivos de falhas de mercado, buscaram-se na literatura especializada subsídios para a elaboração de uma tipologia para os usuários de planos de saúde, atenta à segmentação dos esquemas assistenciais coletivos particulares e privados de financiamento e provisão de serviços no Brasil.

A noção de falha de mercado integra a reflexão de economistas sobre a concorrência perfeita e se refere a situações nas quais a eficiência econômica não pode ser atingida em função das imperfeições dos mecanismos do mercado. De uma maneira muito simplificada, as falhas do mercado, que justificam a intervenção do governo, podem se manifestar tanto na inabilidade do sistema para produzir bens ou serviços que são requeridos quanto na má distribuição de recursos, que poderia ser aperfeiçoada. Os mecanismos de mercado falham quando: 1) custos importantes, como os exemplos clássicos da poluição e da vacinação, não estão refletidos nos preços (externalidades negativas ou positivas); 2) há oligopólio, monopólio; 3) a informação entre compradores e vendedores é desigual (informação assimétrica) (Skipper, 2000).
Da assimetria de informações, ou seja, heterogeneidade do conhecimento sobre os problemas de saúde entre a demanda e a oferta de serviços ou planos de saúde, derivam dois problemas que afetam, intensa e particularmente, a eficiência do mercado de seguros. O primeiro refere-se a uma estratificação dos segurados, segundo a percepção do risco (seleção adversa). Os que sabem que têm baixo risco tenderiam a não procurar cobertura plena e vice-versa. Sob essa acepção, os jovens, por serem saudáveis, demandariam contratos de planos e seguros de saúde com coberturas mais reduzidas do que os idosos, o que por sua vez estimularia a seleção de "bons riscos" por parte das seguradoras (HIAA, 2001).

A segunda fonte geradora de ineficiências é o incremento da exposição aos riscos e uso excessivo de serviços cobertos pelos seguros por parte dos segurados quando existe um terceiro pagador, o governo ou operadoras privadas (moral hazard). Essa constatação respalda as recomendações sobre a utilização dos incentivos positivos, como a redução dos valores das mensalidades dos planos àqueles que abandonarem estilos de vida não saudáveis e os inibidores do consumo, como o co-pagamento e franquias, porque a extensão do moral hazard se ampliaria na medida da não responsabilização do segurado (Pauly e Ramsey, 1999).

Sinteticamente a assimetria de informações se refere ao fato de os pacientes saberem mais sobre sua saúde do que as companhias de seguro e tenderem a usar serviços de saúde mais intensamente se tiverem acesso à cobertura integral (Lisboa e Moreira, 2000).

Os desdobramentos das noções de assimetria de informações para o estudo de casos reais são limitados. Como assinala Teixeira (2001), as curvas de oferta e demanda e seus supostos rígidos destituem as estruturas de seus contextos, de suas determinações históricas e sociais. Benefícios inseridos em sistemas de proteção social articulados pelas empresas empregadoras ou associações de empregados (employer based) baseiam-se na seleção prévia dos segmentos populacionais cobertos. Nesses sistemas, nos quais a variação dos benefícios acompanha a estratificação do mercado de trabalho, as demandas associadas ao status de saúde e a sobreutilização de serviços podem estar atenuadas ou mesmo ausentes.

Portanto, a detecção de evidências sugestivas da presença de seleção adversa e moral hazard requer a elaboração de uma tipologia de cober- 
tura para discriminar as demandas que seriam típicas do livre mercado daquelas originadas diretamente da inserção sociocupacional.

\section{A morfologia do mercado de planos e seguros de saúde no Brasil}

A rede de empresariamento envolvida com a comercialização e oferta de planos de saúde no Brasil é extremamente complexa. Entre as principais lacunas do conhecimento sobre o mercado de assistência suplementar destaca-se a segmentação de suas clientelas. na proliferação de esquemas assistenciais variados quanto aos padrões de financiamento e cobertura dos planos. As distintas estratégias acionadas, desde os anos 40 , pelos setores com maior poder de vocalização de demandas para a obtenção de acesso diferenciado, daquele organizado pela Previdência Social e posteriormente pelo SUS, aos prestadores privados, resultaram na proliferação de esquemas assistenciais variados quanto aos padrões de financiamento e cobertura dos planos.

Os dois grandes segmentos de esquemas assistenciais alternativos ao SUS existentes no Brasil são:

- as redes de serviços organizadas para o atendimento de servidores militares e as instituições de previdência e assistência aos servidores estaduais e municipais administradas por instituições públicas da esfera estadual ou da municipal, tendo como base de financiamento a contribuição obrigatória dos empregados e uma estrutura física constituída por serviços de saúde próprios e/ou hospitais e médicos credenciados;

- os planos e seguros privados de saúde financiados pelas empresas empregadoras e pelas famílias, cuja base física está constituída por hospitais e médicos credenciados e/ou médicos assalariados e hospitais próprios ou ainda por médicos cooperativados, sócios dessas empresas.

Até os meados dos anos 60, a principal e quase exclusiva forma de vinculação aos planos e seguros de saúde foi intermediada pela inserção no mercado formal de trabalho. Ou se estava vinculado aos esquemas assistenciais particulares públicos exclusivos para as forças armadas (FFAA's) e servidores estaduais de determinados estados da Federação ou aos esquemas assistenciais não comerciais, organizados pelas próprias empresas empregadoras e/ou por associações de empregados. Posteriormente, na esteira das políticas previdenciárias privatistas, foram criadas empresas especializadas na comercialização de planos de saúde, as medicinas de grupo e as cooperativas de trabalho médico, representando simultaneamente uma alternativa de externalização das atribuições assistenciais das empresas empregadoras e a perspectiva de constituição de um mercado de planos individuais (Cordeiro, 1984; Oliveira e Fleury-Teixeira, 1978; Viana, 1999).

A partir do final dos anos 80 , a entrada das seguradoras no mercado de planos de saúde e a divulgação de casos de negação de atendimento a pessoas cobertas pelos esquemas privados, contextualizadas pelas mudanças estruturais no mercado de trabalho, imprimiram uma reorganização das demandas e oferta da assistência médica suplementar. A expansão da parcela coberta por planos individuais, constituída pelos segmentos de maior renda, mas com vínculos menos formalizados com o mercado de trabalho, os conflitos entre as empresas de planos de saúde e os prestadores de serviços e a presença das seguradoras no subsegmento não comercial privado de planos de saúde (os planos de autogestão) conferiram intensa visibilidade à magnitude e importância econômica do setor.

Atualmente, ainda estão preservadas, em traços gerais, as clivagens básicas que marcaram a origem do mercado de planos e seguros de saúde no Brasil, quais sejam as formas de contratação dos planos através das empresas empregadoras administradas por instituições públicas ou privadas, ou individualmente, e as modalidades não comerciais e comerciais, que administram os fundos arrecadados e a rede de serviços de saúde. Entretanto, a interpenetração das modalidades comerciais nos denominados planos de autogestão de empresas empregadoras estatais, de órgãos da administração direta da esfera federal e firmas privadas, e também nos esquemas assistenciais particulares públicos (FFAA's e Institutos de Aposentadorias e Pensões de Servidores Estaduais e Municipais) esmaecem os contornos, antes mais definidos, entre os esquemas alternativos.

Adicionalmente, a lógica comercial, voltada à diferenciação do status dos níveis hierárquicos das empresas empregadoras, impôs uma segmentação dos padrões de cobertura. A exceção são os planos de saúde das firmas que adotam políticas de benefícios únicas para todos os empregados e das entidades formadas pelas associações de empregados que ainda se pautam pelo caráter mais mutualista da ação sindical. O restante do mercado de planos e seguros de saú- 
de, independentemente da forma de contratação, está organizado em torno de três tipos de padrões de cobertura. Os serviços considerados excelentes são exclusivos para os portadores dos planos executivos, e aqueles classificados como de terceira linha destinam-se ao atendimento das clientelas vinculadas aos planos básicos. Esse gradiente de acesso aos serviços considerados de menor e maior prestígio/qualidade, sustentado no pagamento diferenciado, por um mesmo procedimento, a médicos e hospitais, mantém as fronteiras entre as clientelas de planos, delineando suas possibilidades de acesso às distintas redes de serviços privados.

\section{Limites e possibilidades das informações da PNAD/98 para o exame da segmentação do mercado de planos e seguros de saúde}

O Suplemento Saúde da PNAD/98 possui dois subconjuntos de perguntas sobre as características dos planos de saúde. O primeiro é conformado por questões relativas ao financiamento dos planos e, portanto, à natureza jurídico-institucional das instituições responsáveis pela contratação das coberturas. O segundo está voltado a explorar as coberturas, considerando variações nos padrões de acesso mediado pelos planos ou seguros de saúde às redes de serviços. Muitas perguntas de ambos os subconjuntos foram dirigidas exclusivamente ao titular do plano.

As respostas referentes ao primeiro grupo de interrogações permitem, a partir da análise e agregação de variáveis originais da PNAD/98, cotejar uma "estratificação passiva" dos segmentos populacionais cobertos (planos empresariais) com uma possível estratificação dos riscos decorrente da demanda ativa por planos e seguros de saúde (planos individuais). Propiciam também uma estimativa mais realista da pouco visível cobertura por planos de saúde administrados diretamente por instituições públicas, que não estão sujeitos, por definição legal, à normatização da Agência Nacional de Saúde Suplementar. Os quesitos incluídos na PNAD/98 para diferenciar a origem institucional e as formas de contratação dos planos são: 1) a vinculação a plano de saúde de empresa ou órgão público, que busca diferenciar os planos particulares públicos (institutos de aposentadorias e pensões dos servidores estaduais e municipais e FFAA's) dos planos privados, mas cuja definição “...destinado a atender servidor público civil da ad- ministração direta, autarquia ou fundação pública federal, estadual e municipal e a seus dependentes, ou a servidor público militar e a seus dependentes...." dá margem a classificá-los como semelhantes; 2) a definição do responsável pelo pagamento da mensalidade do plano de saúde, voltada a diferenciar precipuamente os planos contratados através das empresas empregadoras daqueles adquiridos através de contratos individuais/familiares.

Os resultados iniciais, sempre referidos ao plano único ou ao plano principal considerado pelo entrevistado, apontam a cobertura através de planos de saúde, em 1998, para cerca de 38,8 milhões de pessoas $(24,5 \%$ do total da população), sendo 29 milhões de brasileiros vinculados aos planos privados (75\% do total de cobertos) e os restantes 9,7 milhões associados a planos de saúde de instituições de assistência ao servidor público (federal, estadual ou municipal). Nota-se também o predomínio dos planos empresariais $(60 \%)$ - financiados integral ou parcialmente pelo empregador - em relação aos individuais ( $40 \%$ do total), estes integralmente custeados pelas famílias (IBGE, 2000).

O exame do segundo grupo de características das coberturas dos planos e seguros revela que $89,2 \%$ dos titulares de planos e seguros de saúde dispunham de coberturas através de listas de prestadores de serviços credenciados, enquanto apenas $30,4 \%$ estavam vinculados aos planos que facultam o reembolso. O co-pagamento dos usuários pela utilização dos serviços de saúde estava previsto em $21,4 \%$ dos planos dos titulares cobertos. Esses resultados evidenciam não somente uma superposição do credenciamento com o reembolso para uma pequena parcela dos titulares cobertos, mas, sobretudo, a inequívoca predominância das formas gerenciadas da oferta e demanda de serviços referentes à livre escolha, típica dos seguros privados tradicionais. As coberturas dos planos, segundo a percepção dos entrevistados, não são restritas no que tange ao regime de atendimento e abrangência geográfica. $\mathrm{O}$ atendimento ambulatorial está incluído em $98,6 \%$ dos planos, os exames complementares em $96,3 \%$ e as internações hospitalares em $93,5 \%$. Por outro lado, apenas $31,2 \%$ e $6,6 \%$ dos titulares dos planos declararam estar cobertos por planos que abrangem, respectivamente, a assistência odontológica e o acesso aos medicamentos de uso ambulatorial.

Esse último subconjunto de informações sinaliza padrões mais gerais de coberturas dos 
planos. Depreende-se a presença de um contingente de pessoas vinculadas a planos que seriam mais pródigos em função da previsão do reembolso; e as baixas coberturas para assistência odontológica e medicamentos. Mas, a PNAD/98 é uma fonte limitada para a discriminação da segmentação dos padrões de coberturas, oriundos da diferenciação de status sociocupacional e de renda. Na prática, a diferença entre os planos se concretiza em características das redes de serviços que não foram objeto da pesquisa. $\mathrm{Pa}$ ra complementar os inquéritos populacionais, é necessário desenvolver e divulgar sistematicamente outras bases de informações, cujas unidades de observação - empresas empregadoras e operadoras - sejam mais adequadas ao registro das características dos contratos dos planos e redes de serviços.

O cotejamento dessas informações com outras fontes de dados sobre a assistência médica suplementar, como o cadastro da Agência Nacional de Saúde Suplementar (ANS) e de entidades representativas das operadoras de planos, como a Associação Brasileira de Medicina de Grupo (Abramge) evidencia discrepâncias. Segundo essas últimas fontes, tanto o contingente de pessoas cobertas pelos planos privados quanto a proporção de planos empresariais são maiores do que as estimativas da PNAD/98. Entre as razões que podem justificar tais divergências perfilam-se a categorização demasiado abrangente dos planos de instituições de assistência ao servidor público e as naturais dificuldades para discernir os matizes das diversas formas de financiamento dos planos. É plausível admitir que parte dos entrevistados tenha considerado os descontos em folha de planos empresariais como uma forma de contratação individual, embora as respostas identificassem a co-participação do empregado no pagamento do plano.

Os limites apontados não impedem, porém, o reconhecimento da importância dessas informações. Agregando-se os dois subconjuntos de informações sobre características das coberturas dos planos de saúde delineia-se um panorama muito mais nítido das demandas de planos e seguros de saúde. As informações mais confiáveis e abrangentes estimulam os estudiosos do tema e as instituições governamentais envolvidas com a regulação do mercado de planos e seguros a formular questões mais pormenorizadas, como as que se seguem.

\section{Segmentos sociocupacionais: a estratificação passiva e ativa das demandas por planos de saúde}

O uso de ajustes metodológicos foi necessário ao propósito de definir uma segmentação do mercado de planos e seguros de saúde mais adequada às necessidades de exame da presença de seleção adversa e moral hazard. Em primeiro lugar, procurou-se uma aproximação dos dados da PNAD/98 com o contingente populacional coberto por planos de mercado, isto é, aquele ligado a planos de operadoras sob controle da ANS.

A pergunta da pesquisa sobre a vinculação a planos de instituições de servidores públicos admite respostas positivas de funcionários cobertos por planos de saúde administrados por operadoras de planos privados. Por exemplo, a maior instituição voltada ao atendimento de funcionários públicos federais é a Fundação Grupo Executivo Assistência Patronal (Geap), uma fundação fechada de seguridade social que se inclui entre as operadoras privadas de planos de saúde. Essa também é a situação de outras entidades jurídicas paralelas responsáveis pela administração de planos de saúde de instituições públicas da esfera federal. Para atenuar esse problema de classificação, adotou-se como pressuposto o fato de os servidores civis públicos federais, com exceção dos militares, estarem cobertos por planos de saúde privados. Tal decisão resultou na redução do contingente de pessoas inicialmente consideradas afetas aos planos particulares tidos como públicos, ou seja, o número de indivíduos com planos de instituições de servidores públicos.

Em seguida elaborou-se uma tipologia para ressaltar as implicações decorrentes da origem institucional da demanda à estratificação dos riscos de doença e utilização dos serviços de saúde. Assim, as demandas por planos e seguros de saúde passam a ser reunidas em uma tipologia constituída por cinco categorias, baseada nas características da origem institucional e financiamento das coberturas: 1) Sem plano de saúde; 2) Planos individuais, definidos pelas condições do pagamento do contrato de cobertura ser efetuado diretamente ao plano pelo titular, por outro morador do domicílio ou por pessoa não moradora do domicílio. 3) Planos empresariais particulares públicos, classificados a partir da condição na ocupação ativa ou inativa do titular, que tenha declarado estar coberto por plano de instituição de funcionário público cujo 
vínculo ativo, ou emprego anterior, seja no setor público nas esferas estadual ou municipal ou para os servidores militares nas mesmas condições; 4) Planos empresariais privados, determinados pela conjugação das condições do pagamento do contrato de cobertura ser efetuado pelo titular ou somente pelo empregador, através do emprego atual ou do anterior e da condição de atividade no setor público na esfera federal dos servidores civis e dos empregados no setor privado; 5) Planos de dependentes com titulares fora do domicílio, definidos pelas condições do pagamento do contrato ser efetuado por pessoa não moradora da unidade domiciliar.

Esta última categoria, para a qual as restrições de informações sobre as características do plano são incontornáveis, integra a tipologia por representar um subgrupo populacional diferenciado do restante da população. Os dependentes de titulares que moram em outros domicílios estão concentrados nas faixas etárias mais avançadas ( $45 \%$ de pessoas com mais de 40 anos em contraste com $22 \%$ na população total) e apenas $47 \%$ se declaram sem morbidade, em comparação aos $73 \%$ que se consideram hígidos na população em geral. À primeira vista, este subgrupo apresenta características sugestivas da contratação individual de planos de saúde. Porém, é plausível supor a incidência de diferentes formas institucionais e de contratação em seu interior, pois pelo menos $28 \%$ dos dependentes de titulares que moram em outros domicílios estão vinculados aos planos de instituições de servidores públicos.

Aproximadamente dois milhões de pessoas cobertas por planos de saúde não foram categorizadas por essa tipologia. Tais perdas compreendem cerca de 600 mil pessoas, cobertas por planos financiados através de outras modalidades de pagamento do plano, definidas pelo não enquadramento nos itens anteriores, como o financiamento compartilhado entre todos os moradores, entre moradores e não moradores do domicílio. E ainda a 1,36 milhão de pessoas que não foram classificadas devido a erros e inconsistências relativas às variáveis definidoras das categorias de planos, tais como: falta de resposta de titulares sobre alguma das características dos planos utilizadas para a elaboração da tipologia, tendo como conseqüência a impossibilidade de classificação de seus dependentes; inconsistência dos resultados, como crianças titulares de planos empresariais do setor privado ou empresariais públicos; e ausência de respostas sobre se o plano é ou não de institui- ção de servidor público e a condição e posição na ocupação.

O terceiro ajuste metodológico adotado consistiu na vinculação dos titulares aos dependentes. Uma vez que apenas os titulares responderam a determinadas perguntas sobre as características dos planos, procedeu-se à extensão da tipologia aos dependentes moradores do mesmo domicílio, através da vinculação do código do dependente ao de seu respectivo titular.

A tabela 1 e a figura 1 mostram a segmentação das demandas por planos de saúde e a distribuição das coberturas segundo atividade/inserção no mercado de trabalho. A tipologia utilizada sugere que as proporções das coberturas através dos planos empresariais públicos e por meio de planos individuais são menores do que as informadas pelos dados desagregados. Sob essa tipologia, o mercado privado de planos e seguros de saúde (planos individuais somados aos empresariais privados) conta com pelo menos 27,5 milhões de pessoas, e o empresarial público com 6,5 milhões.

No subsegmento de planos privados (planos empresariais privados somados aos planos individuais), a proporção de planos considerados individual pelos entrevistados é de pelo menos $40 \%$. Entre os cobertos por planos empresariais públicos, $73 \%$ estavam ligados direta ou indiretamente a pessoas com vínculo ativo ao setor público estadual ou municipal, 7\% aos militares, $19 \%$ aos aposentados e o restante a desempregados.

Uma parcela considerável $(34,2 \%)$ de funcionários públicos, vinculados aos planos empresariais, estava coberta por planos privados. É significativa a diferença na quantidade de aposentados cobertos por planos empresariais públicos quando comparada aos que estavam vinculados aos empresariais privados.

A figura 2 expõe a distribuição de cobertura por tipo de plano segundo segmentos sociocupacionais selecionados. Verifica-se o alto percentual de cobertura do subgrupo dos funcionários públicos federais $(68 \%)$ e estaduais e municipais (53\%) por referência ao subgrupo constituído por estudantes, donas de casa, aposentados e pensionistas (26\%). Os segmentos com coberturas proporcionalmente menores do que a do total da população $(24,5 \%)$ são o setor privado (22\%), as crianças $(20,5 \%)$ e os desempregados (17,5\%). O plano individual é mais freqüente entre os inativos (estudantes, donas de casa, aposentados e pensionistas), desempregados. Os planos empresariais privados 
Tabela 1

Distribuição da população coberta segundo tipos de planos - Brasil 1998.

\begin{tabular}{lrrrr}
\hline & \multicolumn{2}{c}{ Condição no Plano } & & Total \\
& Titulares & Dependentes & $\mathrm{n}$ & $\%$ \\
\hline Plano de saúde individual & 6.122 .751 & 5.415 .039 & 11.537 .790 & 29,8 \\
Plano empresarial público & 2.780 .665 & 3.810 .853 & 6.591 .518 & 17,0 \\
militares & 197.385 & 271.599 & 468.984 & 1,2 \\
func. públicos estaduais ou municipais & 1.940 .950 & 2.892 .789 & 4.833 .739 & 12,5 \\
aposentados & 620.655 & 610.205 & 1.230 .860 & 3,2 \\
desempregados & 21.675 & 36.260 & 57.935 & 0,1 \\
Plano empresarial privado & 6.608 .201 & 9.487 .345 & 16.095 .546 & 41,6 \\
func. do setor privado & 5.253 .018 & 7.340 .457 & 12.593 .475 & 32,6 \\
func. públicos federais & 617.166 & 1.114 .062 & 1.731 .228 & 4,5 \\
func. públicos estaduais ou municipais & 405.607 & 634.070 & 1.039 .677 & 2,7 \\
aposentados & 302.574 & 346.792 & 649.366 & 1,7 \\
$\quad$ desempregados & 29.836 & 51.964 & 81.800 & 0,2 \\
Dependentes de titulares fora do domicílio & & 2.491 .729 & 2.491 .729 & 6,4 \\
Não classificados & 724.454 & 1.239 .632 & 1.964 .086 & 5,1 \\
$\quad$ outra forma de contratação do plano & 290.386 & 311.135 & 601.521 & 1,6 \\
indivíduos sem informação completa & 14.012 & 404.370 & 418.382 & 1,1 \\
inconsistência de informação & 420.059 & 524.127 & 944.186 & 2,4 \\
Total & 16.236 .071 & 22.444 .598 & 38.680 .669 & 100 \\
\hline
\end{tabular}

Fonte: PNAD/1998

\section{Figura 1}

Tipos de plano de saúde - Brasil, 1998.

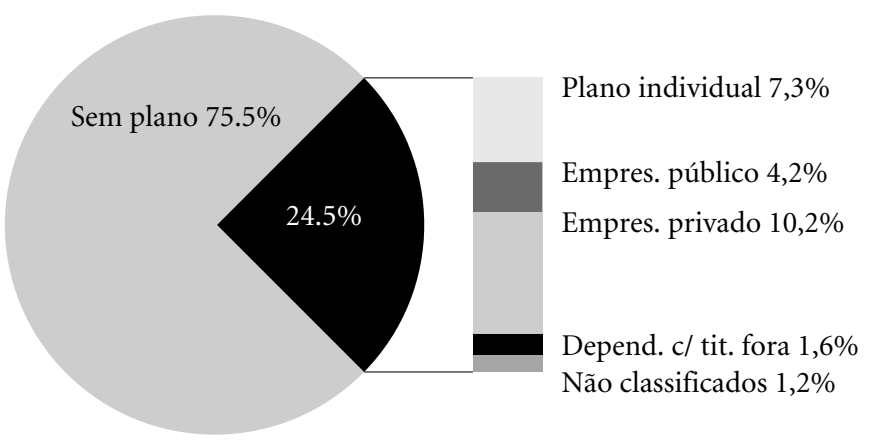


Figura 2

Coberturas através de tipos de planos de saúde segundo grupos sociocupacionais.

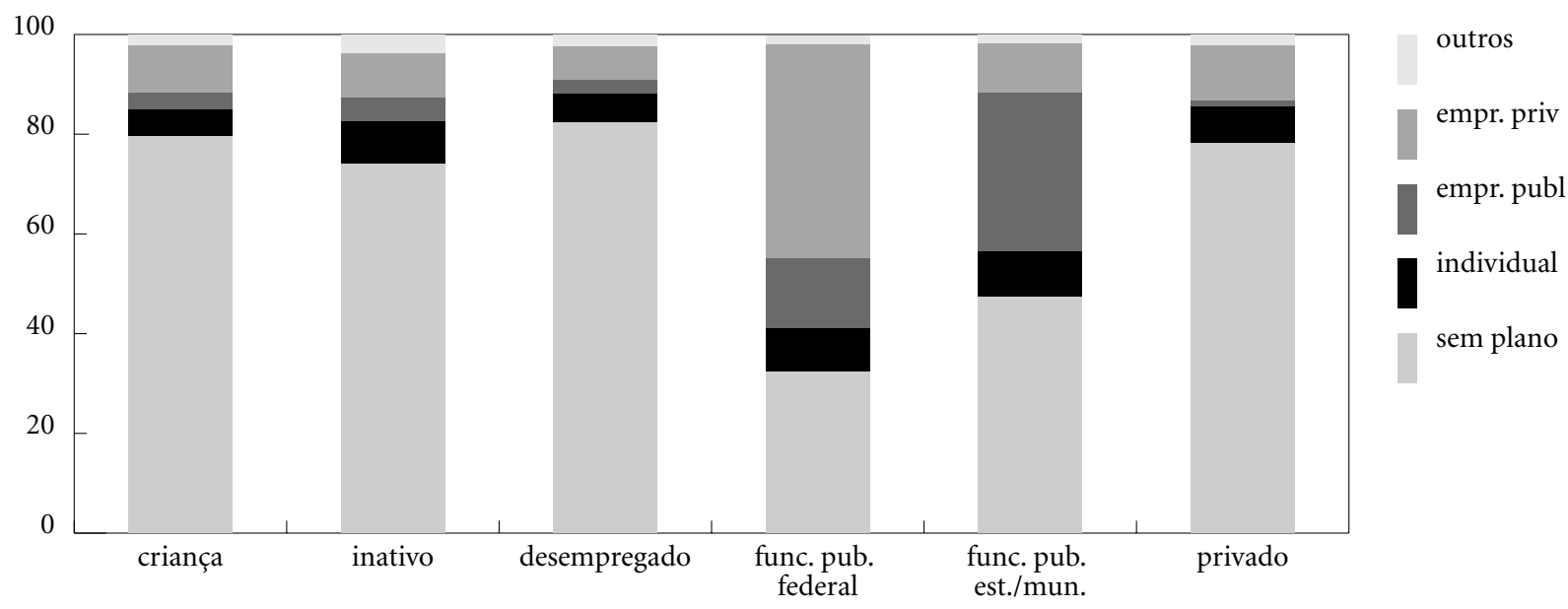

são, em função das categorias utilizadas, a principal alternativa de cobertura para os funcionários públicos federais, para os empregados do setor privado e também para crianças e desempregados.

A distribuição de um conjunto selecionado de variáveis socioeconômicas e regionais segundo a tipologia de planos sugere um gradiente de concentração de pessoas mais velhas nos planos individuais, passando pelo empresarial público e, por último, nos planos empresariais privados. Entre o contingente coberto por planos empresariais privados, observa-se a maior proporção de homens, relativamente aos outros tipos de planos. A proporção de mulheres é maior entre os cobertos pelos planos empresariais públicos. No que diz respeito à renda, há uma hierarquia. As pessoas cobertas pelos planos individuais situam-se na maior faixa de renda $(68,3 \%)$, enquanto essa proporção diminui para 52\% entre os que estavam vinculados aos planos empresariais privados e $47 \%$ no total dos que possuem planos empresariais públicos. Comparado aos planos empresariais, o plano individual concentra os segmentos com maior escolaridade. $\mathrm{O}$ tipo de família formada por casal com filhos menores de 14 anos e as famílias compostas por seis pessoas ou mais é o mais freqüente entre os cobertos pelos planos empresariais. Já o casal sem filhos é mais encontrado nos planos indivi- duais. Os planos empresariais privados e os individuais se concentram na região Sudeste (aproximadamente $65 \%$ ), e os empresariais públicos apresentam uma distribuição mais dispersa entre as regiões. Os segmentos populacionais cobertos por planos de saúde estão concentrados nas regiões metropolitanas ( $47 \%$ do total), onde se encontra apenas $30 \%$ da população residente (Tabela 2).

\section{Estimativas da presença de seleção adversa e moral hazard}

O mercado de planos de saúde no Brasil pode estar sujeito às conseqüências da seleção adversa e moral hazard, que alterariam suas regras de aquisição e utilização, respectivamente. Considerando as informações da figura 2, em combinação com a morfologia de plano de saúde criada, pôde-se suspeitar da existência de ambos os mecanismos. Por outro lado, características individuais e familiares poderiam explicar - pelo menos parcialmente - não só a existência de tais mecanismos, mas também sua magnitude. Na tabela 2 foram levantadas algumas dessas características - julgadas de relevância e disponíveis no banco de dados da PNAD/98 -, verificando-se que a aquisição de plano de saúde (individual) pode depender delas. Além da 
Tabela 2

Distribuição de variáveis socioeconômicas e regionais segundo tipo de plano de saúde - Brasil, 1998.

\begin{tabular}{|c|c|c|c|c|c|c|c|c|}
\hline \multirow{2}{*}{$\begin{array}{l}\text { Variáveis socioeconômicas } \\
\text { e regionais }\end{array}$} & \multicolumn{6}{|c|}{ Tipo de Plano de Saúde (\%) } & \multicolumn{2}{|c|}{ Total } \\
\hline & $\begin{array}{l}\text { Sem plano } \\
\text { de saúde }(\%)\end{array}$ & $\begin{array}{l}\text { Total c/ } \\
\text { plano }\end{array}$ & Individual & $\begin{array}{l}\text { Empres. } \\
\text { público }\end{array}$ & $\begin{array}{l}\text { Empres. } \\
\text { privado }\end{array}$ & Outros* & $\mathrm{N}$ & $\%$ \\
\hline \multicolumn{9}{|l|}{ Grupos etários } \\
\hline 0 a 9 anos & 20,3 & 16,1 & 13,9 & 15,8 & 17,9 & 16,0 & 30.463 .033 & 19,3 \\
\hline 10 a 14 anos & 11,3 & 9,0 & 6,9 & 10,7 & 9,8 & 8,9 & 16.994 .203 & 10,7 \\
\hline 15 a 20 anos & 13,2 & 11,0 & 9,4 & 11,7 & 11,0 & 14,4 & 19.996 .982 & 12,6 \\
\hline 21 a 39 anos & 28,9 & 30,9 & 27,8 & 28,2 & 37,5 & 19,6 & 46.485 .236 & 29,4 \\
\hline 40 a 64 anos & 20,5 & 26,5 & 32,7 & 27,9 & 22,0 & 24,6 & 34.709 .063 & 21,9 \\
\hline 65 anos ou mais & 5,9 & 6,4 & 9,4 & 5,7 & 1,8 & 16,4 & 9.516 .833 & 6,0 \\
\hline \multicolumn{9}{|l|}{ Sexo } \\
\hline masculino & 49,9 & 46,3 & 43,9 & 45,8 & 49,9 & 40,1 & 77.478 .897 & 49,0 \\
\hline feminino & 50,1 & 53,7 & 56,1 & 54,2 & 50,1 & 59,9 & 80.699 .182 & 51,0 \\
\hline \multicolumn{9}{|l|}{ Renda familiar per capita } \\
\hline até $\mathrm{R} \$ 79,99$ & 42,5 & 5,8 & 3,7 & 8,6 & 4,2 & 12,7 & 51.647 .317 & 33,6 \\
\hline de $\mathrm{R} \$ 80,00$ a $\mathrm{R} \$ 119,99$ & 16,0 & 6,6 & 3,5 & 8,6 & 7,5 & 7,9 & 21.086 .396 & 13,7 \\
\hline de $\mathrm{R} \$ 120,00$ a $\mathrm{R} \$ 299,99$ & 29,9 & 32,3 & 24,5 & 35,7 & 35,9 & 34,0 & 46.868 .150 & 30,5 \\
\hline $\mathrm{R} \$ 300,00$ ou mais & 11,5 & 55,4 & 68,3 & 47,2 & 52,4 & 45,4 & 34.029 .407 & 22,2 \\
\hline \multicolumn{9}{|l|}{ Anos de estudo } \\
\hline sem escolaridade & 31,5 & 15,7 & 14,2 & 15,4 & 16,0 & 19,0 & 43.570 .585 & 27,6 \\
\hline 1 a 8 anos & 56,4 & 43,1 & 40,6 & 42,1 & 43,7 & 49,1 & 83.784 .105 & 53,1 \\
\hline 9 a 14 anos & 11,1 & 29,8 & 30,1 & 31,8 & 30,2 & 24,6 & 24.701 .927 & 15,7 \\
\hline 15 anos ou mais & 1,0 & 11,4 & 15,1 & 10,8 & 10,2 & 7,3 & 5.594 .619 & 3,5 \\
\hline \multicolumn{9}{|l|}{ Tipo de família } \\
\hline casal sem filhos & 7,8 & 10,0 & 12,7 & 8,8 & 7,5 & 13,8 & 13.189 .572 & 8,3 \\
\hline casal com filhos $<14$ anos & 53,9 & 49,2 & 40,9 & 52,6 & 60,0 & 26,7 & 83.378 .872 & 52,7 \\
\hline casal com filhos $>14$ anos & 15,6 & 21,9 & 26,8 & 22,1 & 19,9 & 16,0 & 27.057 .691 & 17,1 \\
\hline mãe com filhos $<14$ anos & 8,7 & 5,3 & 4,1 & 4,9 & 3,4 & 16,3 & 12.503 .501 & 7,9 \\
\hline mãe com filhos $>14$ anos & 6,9 & 6,7 & 7,5 & 6,2 & 4,6 & 13,1 & 10.803 .377 & 6,8 \\
\hline outros tipos & 7,2 & 6,9 & 8,0 & 5,4 & 4,6 & 14,1 & 11.245 .066 & 7,1 \\
\hline \multicolumn{9}{|l|}{ Tamanho da família } \\
\hline até 2 pessoas & 14,1 & 17,2 & 21,1 & 14,0 & 11,7 & 31,9 & 23.520 .451 & 14,9 \\
\hline 3 a 5 pessoas & 63,0 & 73,4 & 72,7 & 73,3 & 77,7 & 60,1 & 103.623 .518 & 65,5 \\
\hline 6 pessoas ou mais & 22,9 & 9,4 & 6,3 & 12,7 & 10,6 & 7,9 & 31.034 .110 & 19,6 \\
\hline \multicolumn{9}{|l|}{ Região } \\
\hline Norte & 5,5 & 3,5 & 2,3 & 8,1 & 2,1 & 4,1 & 7.861 .352 & 5,0 \\
\hline Nordeste & 33,7 & 14,7 & 14,1 & 21,8 & 10,9 & 19,3 & 45.918 .899 & 29,0 \\
\hline Sudeste & 38,6 & 59,6 & 65,6 & 41,1 & 64,6 & 52,8 & 69.140 .385 & 43,7 \\
\hline Sul & 15,1 & 15,9 & 13,0 & 18,4 & 16,9 & 15,7 & 24.211 .684 & 15,3 \\
\hline Centro-Oeste & 7,2 & 6,4 & 4,9 & 10,5 & 5,4 & 8,1 & 11.045 .759 & 7,0 \\
\hline \multicolumn{9}{|l|}{ Área } \\
\hline região metropolitana & 25,6 & 47,4 & 50,0 & 36,7 & 50,1 & 46,6 & 48.899 .667 & 30,9 \\
\hline auto-representativo & 19,3 & 27,5 & 27,8 & 27,0 & 27,2 & 28,7 & 33.743 .651 & 21,3 \\
\hline não auto-representativo & 55,1 & 25,1 & 22,2 & 36,2 & 22,7 & 24,7 & 75.534 .761 & 47,8 \\
\hline
\end{tabular}

1) as percentagens referem-se ao total de cada tipo de plano.

2) a variável ocupação aplica-se somente aos maiores de 10 anos.

* Incluídos os dependentes com titular fora do domicílio e aqueles com plano não classificado por falta de informação. 
associação com aquisição de plano de saúde, tais características relacionam-se, também, às condições de saúde das pessoas. Situação análoga ocorre certamente para a utilização de serviços de saúde. Nesse sentido, a complexidade que envolve a deteç̧ão e quantificação dos mecanismos aludidos deve ser analisada, necessariamente, a partir de modelos estatísticos com múltiplos regressores, de forma a incorporar os controles necessários.

Os dados da PNAD/98, ressalvadas as suas limitações metodológicas, permitem explorar, simultaneamente, as relações envolvidas. A principal limitação metodológica refere-se à natureza dos dados. Por serem dados de prevalência, não é possível o estabelecimento de uma relação direta de causa e efeito. Outra limitação importante refere-se aos possíveis vieses envolvidos com as variáveis utilizadas para se avaliarem as condições de saúde. A qualidade dos dados pode afetar não apenas as variáveis de condições de saúde, mas também as co-variáveis exploradas - renda familiar per capita, por exemplo.

A tabela 3 sintetiza os resultados das análises realizadas através de dois modelos logísticos com múltiplos regressores, procurando, assim, contribuir para um melhor entendimento dos mecanismos de seleção adversa e moral hazard. A opção pelo modelo logístico deveu-se ao fato de os dois desfechos considerados serem dicotômicos, e da imediata geração, por esse tipo de modelagem, dos odds ratios (OR) controlados por todas as co-variáveis no modelo.

\section{Seleção adversa}

Para o exame da existência de seleção adversa, foram considerados apenas os indivíduos classificados pela morfologia criada como associados a plano individual. Esse contingente representa quase $30 \%$ do total de indivíduos com plano (tabela 1). Os portadores de plano empresarial foram excluídos, porque esse tipo de plano não se relaciona teoricamente à seleção adversa, uma vez que seus clientes não escolhem as coberturas. A comparação ficou restrita, portanto, entre aqueles com plano individual e aqueles sem plano de saúde.

$\mathrm{Na}$ tabela 3, na coluna intitulada "OR bruto" do modelo para seleção adversa, reproduzem-se, de certa forma, as relações já observadas nas tabelas 1 e 2, através, agora, da medida de associação gerada pelo modelo. Por essa coluna, que retrata associações não ajustadas, as co-variáveis que se relacionam fortemente com a posse de plano de saúde individual são renda familiar per capita, anos de estudo, tamanho da família - inclusive com gradientes - tipo da família, idade e área. Atributos como ocupação, região e sexo, embora também aparentemente relacionados, apresentam medidas de associação menores. Em relação à seleção adversa, não se percebe associação quando avaliada pela escala de mobilidade e pouca relação para morbidade referida e auto-avaliação de saúde. Por outro lado, percebe-se um gradiente para morbidade referida.

Em razão da escala de mobilidade física e da ocupação só se aplicarem aos maiores de 10 anos, foram realizados dois ajustes, sem diferenças marcantes entre eles. Com relação aos efeitos dos ajustes, pode-se ainda dizer que há forte relação de plano de saúde individual com renda e anos de estudo, porém em menor magnitude. A interpretação da variável idade fica prejudicada em conseqüência da exclusão dos planos empresariais, notadamente na idade produtiva. Para o modelo geral, há também um comprometimento da variável "anos de estudo", em função da categoria sem escolaridade incluir as crianças.

Devido à forte dependência da aquisição de plano à renda, a análise foi reavaliada para cada um dos estratos de renda considerados, procurando explorar a noção de interação. Não se observam grandes mudanças nos odds ratios, sugerindo que a seleção adversa não parece acontecer de forma substancialmente diferenciada para as classes de renda consideradas (tabela 4). Diversas outras interações poderiam ser exploradas.

Sintetizando, pode-se dizer que renda e escolaridade são os principais fatores para aquisição de plano de saúde individual. Idade, sexo, localização (região e área) e tamanho da família têm importância residual. Controlando-se por todos esses fatores, o mecanismo de seleção adversa, quando avaliado pela morbidade referida, parece também acontecer de forma residual.

\section{Moral hazard}

O mecanismo de moral hazard foi inicialmente avaliado pelo número mais elevado de consultas médicas no ano, cinco ou mais. Essa opção é questionável, na medida em que a variação no padrão de consumo não pode ser atribuída apenas à mudança de comportamento dos indivíduos, quando segurados por um ou outro tipo de modalidade de plano, mas, sim, 
Tabela 3

Análise da presença de seleção adversa e moral hazard ajustada por regressão logística segundo co-variáveis relevantes - PNAD/98, Brasil.

\begin{tabular}{|c|c|c|c|c|c|c|}
\hline \multirow[t]{2}{*}{ Co-variáveis } & \multicolumn{3}{|c|}{ Modelo 1: seleção adversa* } & \multicolumn{3}{|c|}{ Modelo 2: moral hazard ${ }^{\star *}$} \\
\hline & $\begin{array}{c}\text { OR } \\
\text { bruto }\end{array}$ & $\begin{array}{c}\text { OR } \\
\text { Ajustado }\end{array}$ & $\begin{array}{c}\text { OR aj. } \\
>10 \text { anos }\end{array}$ & $\begin{array}{c}\text { OR } \\
\text { bruto }\end{array}$ & $\begin{array}{c}\text { OR } \\
\text { Ajustado }\end{array}$ & $\begin{array}{c}\text { OR aj. } \\
>10 \text { anos }\end{array}$ \\
\hline \multicolumn{7}{|l|}{ Morbidade referida } \\
\hline dor nas costas ou tendinite & 1,33 & 1,18 & 1,22 & 1,77 & 1,55 & 1,42 \\
\hline $\begin{array}{l}\text { artrite, diabetes, bronquite, } \\
\text { depressão ou tuberculose }\end{array}$ & 1,36 & 1,26 & 1,26 & 3,63 & 2,47 & 2,09 \\
\hline $\begin{array}{l}\text { câncer, doença do coração, } \\
\text { doença renal ou cirrose }\end{array}$ & 1,41 & 1,36 & 1,38 & 6,28 & 3,78 & 3,01 \\
\hline \multicolumn{7}{|l|}{ Auto-avaliação de saúde } \\
\hline boa & 1,25 & 1,03 & 1,07 & 0,25 & 0,33 & 0,41 \\
\hline não boa & 1,00 & & & 1,00 & & \\
\hline \multicolumn{7}{|l|}{ Escala de mobilidade física ${ }^{* * *}$} \\
\hline limitada & 0,88 & & 0,92 & 4,52 & & 1,98 \\
\hline muito limitada & 0,96 & & 0,98 & 6,47 & & 2,62 \\
\hline pouco limitada & 1,03 & & 1,00 & 2,49 & & 1,37 \\
\hline Sem limitação & 1,00 & & & 1,00 & & \\
\hline \multicolumn{7}{|l|}{ Grupos etários } \\
\hline 0 a 9 anos & 0,43 & 1,47 & & 0,45 & 2,10 & \\
\hline 10 a 14 anos & 0,38 & 0,79 & 0,63 & 0,14 & 0,72 & 0,77 \\
\hline 15 a 20 anos & 0,45 & 0,47 & 0,47 & 0,23 & 1,05 & 1,30 \\
\hline 21 a 39 anos & 0,60 & 0,41 & 0,47 & 0,38 & 1,22 & 1,66 \\
\hline 40 a 64 anos & 1,00 & 0,70 & 0,79 & 0,60 & 0,99 & 1,25 \\
\hline 65 anos ou mais & 1,00 & & & 1,00 & & \\
\hline \multicolumn{7}{|l|}{ Sexo } \\
\hline masculino & 1,00 & & & 1,00 & & \\
\hline feminino & 1,27 & 1,36 & 1,32 & 2,12 & 1,91 & 2,13 \\
\hline \multicolumn{7}{|l|}{ Renda familiar per capita } \\
\hline de $\mathrm{R} \$ 80,00$ a $\mathrm{R} \$ 119,99$ & 2,50 & 2,09 & 1,84 & 1,24 & 1,02 & 0,96 \\
\hline de $\mathrm{R} \$ 120,00$ a $\mathrm{R} \$ 299,99$ & 9,45 & 6,64 & 5,29 & 1,54 & 1,02 & 0,95 \\
\hline de $\mathrm{R} \$ 300,00$ ou mais & 68,21 & 33,96 & 24,91 & 1,95 & 1,07 & 0,96 \\
\hline \multicolumn{7}{|l|}{ Anos de estudo } \\
\hline sem escolaridade & 1,00 & & & 1,00 & & \\
\hline 1 a 8 anos & 1,59 & 1,57 & 2,21 & 0,74 & 0,83 & 1,20 \\
\hline 9 a 14 anos & 5,99 & 3,57 & 5,27 & 0,90 & 0,86 & 1,33 \\
\hline 15 anos ou mais & 33,39 & 9,13 & 14,42 & 1,19 & 0,89 & 1,50 \\
\hline \multicolumn{7}{|l|}{ Morfologia de plano de saúde } \\
\hline sem plano de saúde & & & & 1,00 & & \\
\hline plano individual & & & & 2,72 & 2,36 & 2,14 \\
\hline plano empresarial público & & & & 1,72 & 1,78 & 1,55 \\
\hline plano empresarial privado & & & & 2,01 & 2,13 & 2,06 \\
\hline outros & & & & 2,79 & 2,14 & 1,97 \\
\hline \multicolumn{7}{|l|}{ Tipo de família } \\
\hline casal sem filhos & 1,00 & & & & & \\
\hline casal com filhos $<14$ anos & 0,47 & 1,39 & 1,22 & & & \\
\hline casal com filhos $>14$ anos & 1,06 & 1,30 & 1,19 & & & \\
\hline mãe com filhos $<14$ anos & 0,29 & 1,12 & 0,99 & & & \\
\hline mãe com filhos $>14$ anos & 0,67 & 0,83 & 0,79 & & & \\
\hline outros tipos & 0,68 & 0,62 & 0,62 & & & \\
\hline
\end{tabular}


Tabela 3

continuação

\begin{tabular}{|c|c|c|c|c|c|c|}
\hline \multirow[t]{2}{*}{ Co-variáveis } & \multicolumn{3}{|c|}{ Modelo 1: seleção adversa ${ }^{\star}$} & \multicolumn{3}{|c|}{ Modelo 2: moral hazard ${ }^{\star *}$} \\
\hline & $\begin{array}{c}\text { OR } \\
\text { bruto }\end{array}$ & $\begin{array}{c}\text { OR } \\
\text { Ajustado }\end{array}$ & $\begin{array}{l}\text { OR aj. } \\
>10 \text { anos }\end{array}$ & $\begin{array}{c}\text { OR } \\
\text { bruto }\end{array}$ & $\begin{array}{c}\text { OR } \\
\text { Ajustado }\end{array}$ & $\begin{array}{l}\text { OR aj. } \\
>10 \text { anos }\end{array}$ \\
\hline \multicolumn{7}{|l|}{ Tamanho da família } \\
\hline até 2 pessoas & 5,44 & 2,11 & 1,74 & 1,00 & & \\
\hline de 3 a 5 pessoas & 4,21 & 1,79 & 1,59 & 0,64 & 0,90 & 0,90 \\
\hline 6 pessoas ou mais & 1,00 & & & 0,33 & 0,60 & 0,74 \\
\hline \multicolumn{7}{|l|}{ Região } \\
\hline Norte & 0,63 & 0,85 & 0,81 & 0,81 & 0,84 & 0,83 \\
\hline Nordeste & 0,62 & 1,46 & 1,40 & 0,88 & 1,05 & 1,07 \\
\hline Sudeste & 2,51 & 2,02 & 1,94 & 1,43 & 1,36 & 1,35 \\
\hline Sul & 1,27 & 1,14 & 1,16 & 1,30 & 1,34 & 1,35 \\
\hline Centro-Oeste & 1,00 & & & 1,00 & & \\
\hline \multicolumn{7}{|l|}{ Área } \\
\hline região metropolitana & 4,84 & 2,08 & 1,84 & 1,97 & 1,68 & 1,53 \\
\hline auto-representativo & 3,56 & 2,10 & 1,93 & 1,50 & 1,36 & 1,25 \\
\hline não auto-representativo & 1,00 & & & 1,00 & & \\
\hline \multicolumn{7}{|l|}{ Ocupação ${ }^{* * *}$} \\
\hline inativo & 1,20 & & 1,53 & 1,90 & & 1,49 \\
\hline desempregado & 0,73 & & 1,03 & 1,18 & & 1,13 \\
\hline funcionário público federal & 2,82 & & 1,32 & 1,40 & & 1,17 \\
\hline $\begin{array}{l}\text { funcionário público municipal/ } \\
\text { estadual }\end{array}$ & 2,03 & & 0,89 & 1,63 & & 1,26 \\
\hline iniciativa privada & 1,00 & & & 1,00 & & \\
\hline
\end{tabular}

* Desfecho representado por plano individual vs. sem plano.

${ }^{*}$ Desfecho representado por número elevado de consultas (cinco ou mais).

${ }^{* * *}$ Apenas para os maiores de 10 anos.

Tabela 4

Odds ratios, estratificados por renda, da associação entre morbidade referida

e plano de saúde individual, controlados por diversas co-variáveis* - PNAD/98, Brasil.

\begin{tabular}{lcccc}
\hline Morbidade referida $^{* *}$ & \multicolumn{4}{c}{ Renda familiar per capita } \\
& $\begin{array}{c}\mathrm{R} \$ 300,00 \\
\text { ou mais }\end{array}$ & $\begin{array}{c}\text { De } \mathrm{R} \$ 120,00 \\
\text { a R } \$ 299,99\end{array}$ & $\begin{array}{c}\text { De } \mathrm{R} \$ 80,00 \\
\text { a R } \$ 119,99\end{array}$ & $\begin{array}{c}\text { Até } \\
\mathrm{R} \$ 79,99\end{array}$ \\
\hline $\begin{array}{l}\text { dor nas costas ou tendinite } \\
\text { artrite, diabetes, bronquite, }\end{array}$ & 1,27 & 1,17 & 1,04 & 1,00 \\
$\begin{array}{l}\text { depressão ou tuberculose } \\
\begin{array}{l}\text { câncer, doença do coração, } \\
\text { doença renal ou cirrose }\end{array}\end{array}$ & 1,33 & 1,23 & 1,00 & 0,90 \\
\hline
\end{tabular}

* Auto-avaliação de saúde, mobilidade física, idade, anos de estudo, tipo de família,

tamanho da família, região, área e ocupação.

** Referência da associação: sem morbidade referida. 
ao conjunto de fatores envolvidos com a oferta e possibilidades de acesso aos serviços de saúde. O número médio de consultas médicas de 18 países da OECD, em 1987, foi cerca de sete por habitante/ ano. O Japão registrou uma média de 16 consultas por habitante/ano, enquanto na Inglaterra, EUA e Canadá essa relação, no final dos anos 90, atingiu respectivamente 5,4, 5,8 e 6,4 consultas/habitante/ano (www.oecd.observer. org). Portanto, o parâmetro selecionado não pode ser tomado como representativo de um "excesso" de consumo. O que daqui por diante se define como moral hazard, para fins de diálogo com a literatura especializada, deve ser considerado, no caso brasileiro, apenas um padrão de consumo, menos sujeito às agruras antepostas para o acesso aos serviços da rede SUS.

Pelos valores observados na tabela 3 , as três variáveis relacionadas às condições de saúde mostraram-se associadas à utilização de mais de cinco consultas médicas, diminuindo porém de magnitude nos modelos ajustados. A renda familiar, que inicialmente parecia relacionada, perde sua associação quando controlada por outros fatores. O sexo feminino, mesmo quando ajustado, mantém sua maior chance de consultas elevadas e a escolaridade passa a apresentar um pequeno gradiente, com indivíduos com mais anos de estudo procurando mais consultas.

Especificamente relacionada ao moral hazard, a variável morfologia de plano de saúde aponta para a sua existência, podendo-se estimar que é duas vezes maior a chance de um número elevado de consultas entre aqueles que têm plano em comparação aos não cobertos (tabela 3). Deve-se observar também que praticamente não há diferença entre os diversos tipos de plano. Isto é, plano individual não parece estimular o consumo mais elevado de serviços do que os planos empresariais.

Uma outra forma de se avaliar a presença de mecanismo de moral hazard é por meio dos gastos com saúde. A variação das despesas com saúde, embora devam ser consideradas as advertências a respeito das limitações da PNAD frente à Pesquisa de Orçamento Familiar (POF) (Barros et al., 1997), pode expressar diferentes padrões de gastos das famílias para um consumo razoavelmente uniforme de serviços. Observa-se que as chances ajustadas de os indivíduos cobertos por planos de saúde utilizarem mais que cinco consultas em relação aos sem planos variam entre o máximo de 2,14 para a categoria plano individual e o mínimo de 1,78 vezes para os que estavam vinculados aos planos empresariais públicos. Já os gastos médios mensais com saúde, excetuando-se os próprios planos, quando não ajustados, parecem expressar dois padrões diferenciados. O gasto médio mensal de quem declarou cobertura de plano tipo individual ou era dependente de titular que mora em outro domicílio se situa em torno de $\mathrm{R} \$ 74,00$ e $\mathrm{R} \$ 93,00$, respectivamente. Os que estavam vinculados aos planos empresariais (público e privado) referiram um gasto em torno de $\mathrm{R} \$ 52,00$ e $\mathrm{R} \$ 58,00$, mais próximos aos $\mathrm{R} \$ 54,00$ efetuados por quem não tem plano.

A tabela 5 sintetiza o resultado de um modelo de regressão linear múltipla para o logaritmo dos gastos mensais com saúde, excluindo-

Tabela 5

Análise da presença de moral hazard ajustada por regressão linear segundo co-variáveis relevantes - PNAD/98, Brasil.

\begin{tabular}{|c|c|c|}
\hline Co-variáveis & coef. & efeito $\%$ \\
\hline \multicolumn{3}{|l|}{ Morbidade referida } \\
\hline dor nas costas ou tendinite & 0,020 & 2,0 \\
\hline $\begin{array}{l}\text { artrite, diabetes, bronquite, } \\
\text { depressão ou tuberculose }\end{array}$ & 0,044 & 4,5 \\
\hline $\begin{array}{l}\text { câncer, doença do coração, } \\
\text { doença renal ou cirrose }\end{array}$ & 0,243 & 27,5 \\
\hline \multicolumn{3}{|l|}{ Auto-avaliação de saúde } \\
\hline não boa & 0,000 & \\
\hline boa & $-0,149$ & $-13,8$ \\
\hline \multicolumn{3}{|l|}{ Escala de mobilidade física ${ }^{\star}$} \\
\hline muito limitada & 0,319 & 37,6 \\
\hline limitada & 0,161 & 17,5 \\
\hline pouco limitada & 0,030 & 3,0 \\
\hline sem limitação de mobilidade & 0,000 & \\
\hline \multicolumn{3}{|l|}{ Grupos etários } \\
\hline de 10 a 14 anos & 0,000 & \\
\hline de 15 a 20 anos & $-0,102$ & $-9,7$ \\
\hline de 21 a 39 anos & $-0,304$ & $-26,2$ \\
\hline de 40 a 64 anos & $-0,213$ & $-19,2$ \\
\hline 65 anos ou mais & 0,092 & 9,6 \\
\hline \multicolumn{3}{|l|}{ Sexo } \\
\hline masculino & 0,000 & \\
\hline feminino & $-0,189$ & $-17,2$ \\
\hline \multicolumn{3}{|l|}{ Renda familiar per capita } \\
\hline de $\mathrm{R} \$ 80,00$ a $\mathrm{R} \$ 119,99$ & 0,155 & 16,8 \\
\hline de $\mathrm{R} \$ 120,00$ a $\mathrm{R} \$ 299,99$ & 0,278 & 32,0 \\
\hline $\mathrm{R} \$ 300,00$ ou mais & 0,606 & 83,3 \\
\hline
\end{tabular}

(continua) 
Tabela 5

continuação

\begin{tabular}{|c|c|c|}
\hline Co-variáveis & coef. & efeito $\%$ \\
\hline \multicolumn{3}{|l|}{ Anos de estudo } \\
\hline sem escolaridade & 0,000 & \\
\hline de 1 a 8 anos & 0,221 & 24,7 \\
\hline de 9 a 14 anos & 0,420 & 52,2 \\
\hline de 15 anos ou mais & 0,673 & 96,0 \\
\hline \multicolumn{3}{|l|}{ Morfologia de planos de saúde } \\
\hline sem planos & 0,000 & \\
\hline individual & 0,263 & 30,1 \\
\hline empresa pública & 0,007 & 0,7 \\
\hline empresa privada & 0,145 & 15,6 \\
\hline não classificado & 0,157 & 17,0 \\
\hline $\begin{array}{l}\text { dependente com titular fora } \\
\text { do domicílio }\end{array}$ & 0,284 & 32,8 \\
\hline \multicolumn{3}{|l|}{ Tamanho da família } \\
\hline até 2 pessoas & 0,000 & \\
\hline de 3 a 5 pessoas & 0,079 & 8,2 \\
\hline de 6 pessoas ou mais & 0,118 & 12,5 \\
\hline \multicolumn{3}{|l|}{ Região } \\
\hline Nordeste & 0,000 & \\
\hline Norte & 0,120 & 12,7 \\
\hline Centro-Oeste & 0,307 & 35,9 \\
\hline Sudeste & 0,261 & 29,8 \\
\hline Sul & 0,097 & 10,2 \\
\hline \multicolumn{3}{|l|}{ Área ${ }^{\star *}$} \\
\hline região metropolitana & 0,017 & 1,7 \\
\hline auto-representativo & 0,030 & 3,0 \\
\hline não auto-representativo & 0,000 & \\
\hline
\end{tabular}

1) intercepto $=2,796$

2) R2 ajustado $=0,169$ tal. Com relação à variável renda, embora esta não esteja associada a um número elevado de consultas, observa-se um gradiente com relação aos gastos com saúde.

A última coluna da tabela 5 ilustra, para cada co-variável, a variação percentual nos gastos de saúde em relação à categoria de referência (valor 0). Entretanto, não fica explícito o valor esperado dos gastos com saúde. Para melhor ilustrar as evidências de moral hazard advindas da nossa análise, a tabela 6 apresenta os gastos esperados para as combinações de morfologia de plano e morbidade referida para um estrato populacional definido por mulheres com boa auto-avaliação de saúde, sem limitação de mobilidade, com pelo menos 65 anos, com renda familiar superior a $\mathrm{R} \$ 300,00$, com 9 a 14 anos de estudo, em famílias entre 3 e 5 pessoas, na região metropolitana da região Sudeste. Esta última tabela mostra que as mulheres com plano individual, no estrato considerado, ou as que são dependentes de titulares que moram em outros domicílios, gastam mais com saúde para todas as categorias de morbidade referida, sustentando a hipótese da presença do mecanismo de moral hazard.

\section{Considerações finais}

A menção aos mecanismos de assimetria de informações no mercado de planos e seguros privados de saúde vem acompanhada pelas recomendações da oferta de coberturas parciais e incremento do co-pagamento. A tendência, por parte das empresas de planos e seguros de saúde, de evitar a cobertura de indivíduos com alto risco de adoecer (cream skimming) explicita a necessidade da regulação governamental (Greenberg, 1998). Entre outras medidas de gerenciamento dos cuidados à saúde (managed care), preconiza-se a ampliação do conhecimento sobre as condições de saúde dos clientes como antídoto à escalada do uso e custo dos serviços de saúde. Embora largamente difundido, esse receituário vem sendo debatido sob a ótica conceitual e empírica.

Shmanske (1996) considera que a noção de assimetria de informações tomada como justificativa à intervenção governamental ofusca o tema fundamental da economia - a escassez de recursos. Se os recursos são escassos, o melhor que a sociedade pode fazer é deixar ao mercado a tarefa de equilibrar custos e benefícios. Sob essa acepção, nos serviços de saúde, a informa- 
Tabela 6

Gastos médios esperados, em reais, segundo morbidade referida e morfologia de plano, para mulheres com boa auto-avaliação de saúde, sem limitação de mobilidade, com 65 anos de idade ou mais, com renda familiar per capita superior a $\mathrm{R} \$ 300,00$, com 9 a 14 anos de estudo, em famílias entre 3 e 5 pessoas, na região metropolitana da região Sudeste - PNAD/98, Brasil

\begin{tabular}{|c|c|c|c|c|}
\hline \multirow[t]{2}{*}{ Morfologia } & \multicolumn{4}{|c|}{ Morbidade referida* } \\
\hline & 0 & 1 & 2 & 3 \\
\hline Sem plano & $\mathrm{R} \$ 51,06$ & $\mathrm{R} \$ 52,09$ & $\mathrm{R} \$ 53,36$ & $\mathrm{R} \$ 65,10$ \\
\hline Individual & $\mathrm{R} \$ 66,42$ & $\mathrm{R} \$ 67,76$ & $\mathrm{R} \$ 69,41$ & $\mathrm{R} \$ 84,69$ \\
\hline Empresarial público & $\mathrm{R} \$ 51,42$ & $\mathrm{R} \$ 52,46$ & $\mathrm{R} \$ 53,73$ & $\mathrm{R} \$ 65,56$ \\
\hline Empresarial privado & $\mathrm{R} \$ 59,03$ & $\mathrm{R} \$ 60,22$ & $\mathrm{R} \$ 61,68$ & $\mathrm{R} \$ 75,26$ \\
\hline Não classificados & $\mathrm{R} \$ 59,74$ & $\mathrm{R} \$ 60,95$ & $\mathrm{R} \$ 62,43$ & $\mathrm{R} \$ 76,17$ \\
\hline Depend. com tit. fora & $\mathrm{R} \$ 67,83$ & $\mathrm{R} \$ 69,20$ & $\mathrm{R} \$ 70,88$ & $\mathrm{R} \$ 86,49$ \\
\hline
\end{tabular}

Modelo linear ajustado para o logaritmo dos gastos com saúde, excluídos os gastos com planos de saúde. * $0=$ sem morbidade referida; $1=$ dor nas costas ou tendinite; 2 = artrite, diabetes, bronquite, depressão ou tuberculose; 3 = câncer, doença do coração, doença renal ou cirrose.

ção assimétrica surge porque a obtenção e a transmissão de informação fidedigna é cara e as políticas governamentais tentam mascarar esses custos.

Em outra direção, estudiosos como Cardon e Hendel (2001) não encontram evidências da presença de assimetria de informações, após a análise de informações de um inquérito nacional sobre despesas médicas, e refletem sobre a interveniência das empresas empregadoras de grande porte na determinação da amplitude das coberturas e preços dos valores das mensalidades dos planos.

No Brasil, verifica-se uma "seleção favorável” dos riscos no segmento vinculado aos planos de saúde. De acordo com as informações da PNAD/98, a proporção de pessoas vinculadas aos planos de saúde que consideram seu estado de saúde muito bom $(28,5 \%)$ é mais alta do que a do total de cobertos $(24,5)$ na população em geral. No segmento vinculado apenas ao SUS apenas $71,5 \%$ declararam um estado de saúde muito bom, relativamente ao total de $75,5 \%$ da população coberta exclusivamente por essa modalidade de proteção de saúde.

Segundo a mesma fonte de informações, a utilização de serviços de saúde é muito mais intensa no segmento coberto por planos de saúde, como se depreende pela diferença entre a proporção de pessoas com plano (72\%) que passaram por ao menos uma consulta médica, nos 12 meses antes da entrevista, relativamente aos $41 \%$ do total do segmento coberto exclusivamente pelo SUS. As internações hospitalares para o segmento coberto por planos de saúde $(10,5 \%)$ também foram mais acessíveis do que as referidas pelos entrevistados sem plano $(9,2 \%)$.

Um estudo recente sobre o status de saúde e o padrão de consumo de serviços, para a população com idade acima de 60 anos em uma cidade de pequeno porte, evidenciou padrões diferenciados entre os cobertos por planos de saúde e os que são exclusivamente atendidos pelos serviços públicos. Quem estava vinculado a plano de saúde, apesar de relatar melhores condições de saúde, visitou mais o médico e usou mais intensamente os medicamentos prescritos (Lima-Costa et al., 2002). Por outro lado, resultados de um inquérito populacional realizado entre 1989 e 1990 na região Sudeste da área metropolitana de São Paulo mostraram uma concentração de cobertura de planos de saúde nas faixas etárias correspondentes à população economicamente ativa. Entre a população mais idosa (50 anos ou mais), 78\% dos homens e $80 \%$ das mulheres não tinham cobertura de planos de saúde (Cesar e Tanaka, 1996).

A análise dos dados da PNAD/98, voltada para a busca de evidências empíricas das falhas de mercado, não é conclusiva. Mas é inquestionável a dissociação entre as condições de saúde e o uso de serviços e os gastos com saúde, isto é, o predomínio da adoção dos critérios contributivos como definidores do uso e acesso aos serviços de saúde sobre os clínico-epidemiológicos.

Portanto, as iniquiidades presentes no sistema de saúde brasileiro nos desafiam a adotar políticas públicas direcionadas a uma regulação 
voltada à ampliação do direito à saúde. O aprofundamento do conhecimento sobre a dinâmica do mercado de planos e seguros de saúde, certamente, contribui para explicitar tal prioridade.

\section{Referências bibliográficas}

Barros RP, Mendonça R \& Neri M 1997. An evaluation of the measurement of income and expenditure in household surveys: POF versus PNAD. Anais do XVII Encontro Brasileiro de Econometria, Salvador.

Cardon JH \& Hendel I 2001. Asymmetric information in health insurance: evidence from the National Medical Expenditure Survey. RAND Journal of Economics v. 32 (3): 408-427.

Cesar CLG \& Tanaka OU 1996. Inquérito domiciliar como instrumento de avaliação de serviços de saúde: um estudo de caso na região Sudoeste da área metropolitana de São Paulo, 1989-1990. Caderno de Saúde Pública 12(2): 59-70.

Cordeiro H 1984. As empresas médicas: as transformações capitalistas da prática médica. Edições Graal, Rio de Janeiro.

Greenberg W 1998. The health care marketplace. Health Administration Press, Chicago.

HIAA - Health Insurance Association of America 2001. Source book of insurance data 1999-2000. HIAA, Washington.

Instituto Brasileiro de Geografia e Estatística - IBGE 2000. Acesso e utilização de serviços de saúde 1998. Rio de Janeiro.

Lima-Costa MF et al. 2002. Projeto Bambuí: plano privado de saúde e utilização de serviços pela população idosa. Caderno de Saúde Pública 18 (1): 177-186.
Lisboa MB \& Moreira H 2000. Should we be afraid of managed care? A theoretical assessment. Texto para Discussão no 413. Departamento de Economia PUCRio, Rio de Janeiro.

Pauly MV \& Ramsey SD 1999. Would you like suspenders to go with that belt? An analysis of optimal combinations of cost sharing and managed care. Journal of Health Ecomics 18: 443-458.

Oliveira JA \& Fleury Teixeira SM 1978. Medicina de grupo: a medicina e a fábrica. In Guimarães R (org.). Saúde e medicina no Brasil. Graal, Rio de Janeiro.

Skipper Jr HD 2002. International risk and insurance: an environmental-manegerial approach. Irwin McGrawHill, Boston.

Teixeira A 2001. Mercado e imperfeições de mercado. $\mathrm{Ca}$ derno de Saúde Suplementar (II Ciclo de Oficinas). Agência Nacional de Saúde Suplementar, Rio de Janeiro.

Viana DAL 1999. O papel do IAMSPE e o modelo de seguro social. In Junqueira L (org.). Reforma do Estado e mudança institucional: o caso do IAMSPE. Edições Fundap, São Paulo.

Artigo apresentado em 25/3/2002

Aprovado em 11/4/2002

Versão final apresentada em17/4/2002 